\title{
Artéria femoral profunda: uma opção como origem de fluxo para derivações infrageniculares
}

\author{
Deep femoral artery: an option as inflow site \\ in infragenicular bypasses
}

\begin{abstract}
Francisco Cardoso Brochado Neto ${ }^{1}$, Ivan Benaduce Casella ${ }^{1}$, Marcelo Fernando Matielo ${ }^{2}$, Tulia Brasil Simões ${ }^{3}$, Arnaldo Rolim Ricartte ${ }^{3}$, Ricardo Lacerda ${ }^{3}$, Luiz Carlos Bergamo ${ }^{4}$, Roberto Sacilotto 5
\end{abstract}

\section{Resumo}

Contexto: $\mathrm{Na}$ isquemia crítica, a artéria femoral profunda pode tornar-se a opção mais distal como origem de fluxo para derivações distais em casos de oclusão da origem da artéria femoral superficial associada a prega inguinal hostil.

Objetivo: Avaliar, retrospectivamente, a artéria femoral profunda como doadora de fluxo para derivações infrageniculares.

Métodos: De 2000 a 2005, 129 derivações infrageniculares apresentaram anastomose proximal nas artérias femorais, comum (40), superficial (72) e profunda (17). O presente estudo teve como foco a artéria femoral profunda, e suas indicações foram: prega inguinal hostil (seis casos), limite da extensão do substituto (seis casos) e ambos os fatores (outros cinco casos). Foram abordadas a primeira e a segunda porção em 12 casos e a terceira porção em cinco casos. As cirurgias foram secundárias em $47 \%$ dos casos, e os substitutos utilizados foram veias do membro superior em 11 casos, safena interna em cinco e safena externa em um caso.

Resultados: No total dos enxertos (129), as estimativas de perviedade primária e salvamento do membro foram: $68,0 \%$ e $84,7 \%$, respectivamente, com erro padrão (EP) aceitável $(0,1)$ em 36 meses. Quando o grupo foi estratificado, as artérias femorais comum, superficial e profunda apresentaram resultados comparáveis de perviedade primária $(63,3,70,2$ e $64,7 \% ; \mathrm{p}=0,63)$ e salvamento do membro $(83,1,82,4$ e $92,3 \% ; p=0,78)$. A perviedade dos enxertos com origem nas porções proximal e distal da artéria femoral profunda, bem como das cirurgias primárias e secundárias, foram comparáveis, sem diferença estatística significante $(\mathrm{p}=0,89$ e $\mathrm{p}=0,77$, respectivamente).

Conclusão: A artéria femoral profunda mostrou ser acessível e efetiva como origem de fluxo de enxertos infrageniculares, com resultados satisfatórios de perviedade e salvamento do membro.

Palavras-chave: Artéria femoral profunda, anastomose proximal, derivações infrageniculares.

\begin{abstract}
Background: Deep femoral artery can be the most distal technical option as donor site in patients with critical limb ischemia presenting superficial artery occlusion and hostile groins.
\end{abstract}

Objective: To retrospectively assess the deep femoral artery as an inflow site for infragenicular bypass grafts.

Methods: From 2000 to 2005, 129 infragenicular bypass grafts with proximal anastomosis located in femoral arteries were performed. Forty were located in the common femoral artery (CFA), 72 in the superficial femoral artery (SFA) and 17 in the deep femoral artery (DFA). Indications for using the DFA as inflow were hostile groin (six cases), limited arterial substitute length (six cases) or both (five cases). Anastomosis site was located in the first or second portion in 12 cases, and in the third in five cases. The surgery was secondary in $47 \%$ of the cases, and the arterial substitutes used were arm veins (11), greater saphenous vein (five) and lesser saphenous vein (one).

Results: Primary patency and limb salvage rates were 68.0 and $84.7 \%$, respectively, with acceptable standard error (0.1) in 36 months. The results of patency divided by inflow artery were similar (CFA, $63.3 \%$; SFA, 70.2\%; DFA 64.7\%; $\mathrm{p}=0.63$ ), as well as limb salvage rates (CFA, $83.1 \%$; SFA, $82.4 \%$; DFA $92.3 \%$; $=0.78$ ). Analyzing the deep femoral group, no difference of patency rates was observed when the anastomotic site was compared (proximal vs. distal portions of the DFA) or between patients with or without previous grafts. ( $\mathrm{p}=$ 0.89 and 0.77 , respectively).

Conclusion: Deep femoral artery is a feasible and effective option as donor site for infragenicular bypass grafts, with satisfactory patency and limb salvage rates.

Keywords: Deep femoral artery, proximal anastomosis, infragenicular bypass grafts.

1. Médico assistente e preceptor de residentes, Serviço de Cirurgia Vascular, Hospital do Servidor Público Estadual de São Paulo (HSPESP), São Paulo, SP.

2. Médico assistente e responsável, Setor de Enfermaria, Serviço de Cirurgia Vascular, HSPE-SP, São Paulo, SP.

3. Ex-residente, Serviço de Cirurgia Vascular, HSPE-SP, São Paulo, SP.

4. Médico. Chefe, Serviço de Cirurgia Vascular, HSPE-SP, São Paulo, SP.

5. Diretor, Serviço de Cirurgia Vascular, HSPE-SP, São Paulo, SP.

Não foram declarados conflitos de interesse associados à publicação deste artigo.

Artigo submetido em 11.10.07, aceito em 19.05.08.

J Vasc Bras. 2008;7(3):193-202.

Copyright@ 2008 by Sociedade Brasileira de Angiologia e de Cirurgia Vascular 


\section{Introdução}

O uso preferencial da artéria femoral comum (AFC) como origem de fluxo de enxertos infra-inguinais começou a perder força após publicações como as de Veith et al. ${ }^{1}$, Mills et al. ${ }^{2}$ e, mais recentemente, Ballota et al. ${ }^{3}$, em estudo randomizado, que utilizaram outras artérias, como as femorais, superficial (AFS) e profunda (AFP), sem doença proximal, e alcançaram resultados de função dos enxertos equivalentes aos da AFC.

Alguns pacientes com oclusão do segmento femoropoplíteo necessitam de reoperação em prega inguinal hostil ${ }^{4}$, na presença ou não de infecção, em condições desfavoráveis para o acesso anterior convencional para a AFC. Outros apresentam limitação do substituto, na falta da veia safena interna, provocando o emprego de substitutos alternativos de menor extensão como as veias do membro superior (VMS) ${ }^{5}$ ou safena externa ${ }^{6}$. Nessas situações, a AFP pode aparecer como opção mais distal e preservada para a anastomose proximal de enxertos. Neste estudo, avaliamos retrospectivamente a AFP como doadora de fluxo para derivações infrageniculares.

\section{Método}

De janeiro de 2000 a dezembro de 2005, no Hospital do Servidor Público Estadual de São Paulo, foram analisadas retrospectivamente 129 derivações infrageniculares de um total de 497 revascularizações infra-inguinais. Foram selecionadas, para o estudo, derivações com as seguintes características: origem nas artérias femorais comum, superficial e profunda; substitutos autógenos; e artérias receptoras infrageniculares. Os dados clínicos e demográficos dos pacientes estudados estão resumidos na Tabela 1.

Nas 129 derivações, as artérias doadoras utilizadas foram as femorais: superficial em 72 casos $(55,8 \%)$, comum em $40(31,0 \%)$ e profunda em $17(13,2 \%)$. Não

Tabela 1 - Dados demográficos e clínicos

\begin{tabular}{|c|c|c|}
\hline Variáveis & n $(\%)$ & Média (EP) \\
\hline Idade & & $70,2 \pm 8,5$ \\
\hline \multicolumn{3}{|l|}{ Sexo } \\
\hline Homem & $81(62,8)$ & \\
\hline Mulher & $48(37,2)$ & \\
\hline Hipertensão arterial & $98(76,0)$ & \\
\hline Diabetes melito & $80(62,0)$ & \\
\hline Diabetes melito insulino-dependente & $30(23,3)$ & \\
\hline Insuficiência cardíaca congestiva & $27(20,9)$ & \\
\hline Doença coronariana & $27(20,9)$ & \\
\hline Tabagismo & $57(44,2)$ & \\
\hline Doença renal & $27(20,9)$ & \\
\hline \multicolumn{3}{|l|}{ Quadro clínico } \\
\hline Claudicação severa & $06(4,7)$ & \\
\hline Dor de repouso & $19(14,7)$ & \\
\hline Lesão trófica & $103(79,8)$ & \\
\hline $\begin{array}{l}\text { Assintomático (aneurisma de } \\
\text { poplítea) }\end{array}$ & $1(0,8)$ & \\
\hline Seguimento & & $23,8 \pm 18,7$ \\
\hline
\end{tabular}

$\mathrm{EP}=$ erro padrão. 
houve diferença estatística significante entre os subgrupos quanto à presença das variáveis demográficas e clínicas. As artérias receptoras mais utilizadas foram a poplítea infragenicular, fibular e tibiais. Os substitutos foram autógenos em todos os casos, sendo as veias safena interna utilizada em 108 deles, safena externa em dois, membro superior em 16 e composição de veias safena interna e do membro superior em três casos. A técnica de implante das veias foi, preferencialmente, à maneira removida, não-reversa e devalvulada, utilizando o valvulótomo de Mills em 96 casos, e reversa em 32. Em um caso, utilizou-se a técnica in situ, conforme mostra a Tabela 2. As indicações para o uso da AFP como origem de fluxo foram: prega inguinal hostil em seis dos 17 casos; limitação da extensão do substituto em seis casos; e presença dos dois fatores nos cinco restantes. A AFP foi dividida em três zonas: a proximal, que se estende da sua origem até a artéria circunflexa lateral do fêmur; a média, que se estende da artéria circunflexa lateral do fêmur até o segundo ramo perfurante e entra no triângulo femoral; e a distal, após o segundo ramo perfurante ${ }^{7}$. As anastomoses proximais dos enxertos foram feitas nas porções proximal e média em 12 casos, e na porção distal em cinco casos. O uso da terceira porção (distal) da AFP foi resultado da impossibilidade de acesso aos segmentos proximais da artéria.

As artérias receptoras das derivações com origem na AFP foram: poplítea em cinco casos, tibial anterior em cinco, tibial posterior em três (Figura 1), fibular em dois, genicular sural em um e enxerto anterior (femoral superior - troncotibiofibular) em um caso. Das 17 derivações da AFP, oito (47\%) foram cirurgias secundárias, sendo seis por enxerto distal ocluído, uma por infecção de ferida operatória pós-trombectomia e uma para evitar exposição de prótese aorto-femoral. A maioria dos substitutos foram veias de braço (11 casos): veia basílica em três casos, veia cefálica em quatro e alça basílicocefálica em quatro, todas implantadas em artérias de perna. Os substitutos restantes foram veia safena interna em cinco casos e safena externa em um. Dezesseis veias foram colocadas de maneira removida e devalvulada e uma reversa. As cirurgias foram realizadas com magnificação de imagem (lupa 3x).

O estudo arteriográfico da artéria femoral profunda foi, preferencialmente, feito em projeções anteroposterior e oblíqua.
A porção da AFP utilizada como fonte do enxerto foi escolhida obedecendo ao critério técnico de doença proximal ausente ou estenose de no máximo $20 \%$ da $1 \mathrm{uz}^{2,3}$, priorizando sempre que possível as porções iniciais.

Para abordar a primeira e segunda porções da AFP, a preferência foi o acesso ântero-medial para cirurgias primárias, realizado medialmente ao músculo sartório. Nos casos com prega inguinal hostil, foi realizado o acesso ântero-lateral, lateralmente ao músculo sartório (Figura 2).

Para a terceira porção, foi utilizado o acesso pósteromedial da coxa por uma incisão longitudinal feita entre os músculos adutor longo e grácil. O músculo adutor longo é deslocado superiormente, e os músculos adutor curto e grande adutor são deslocados inferiormente, dessa maneira permitindo a exposição da AFP, que pode então ser isolada abaixo do segundo ramo perfurante, na sua porção distal ${ }^{8}$ (Figura 2).

Todos os enxertos entraram no programa de vigilância de enxertos autógenos. A análise dos resultados foi feita pela life table (Kaplan-Meier), e a comparação dos resultados, pelo teste de log rank, com o programa Statistical Package for the Social Sciences (SPSS), versão 10.0 .

\section{Resultados}

Os pacientes das 129 derivações foram acompanhados durante 36 meses, com seguimento médio de $23,8 \pm 18,7$ meses. No grupo total, ocorreram 34 oclusões de enxertos e 18 grandes amputações, eventos que provocaram estimativas de perviedade primária de $68,0 \%$ e de salvamento de membro de $84,7 \%$, com erro padrão (EP) aceitável menor que $10 \%$. As estimativas de perviedade primária foram estratificadas conforme o sítio da anastomose proximal, e os resultados encontrados foram de $63,3 \%$ para a AFC, $70,2 \%$ para a femoral superficial e $64,7 \%$ para a femoral profunda, com EP $=11 \%$, mas sem diferença estatística significativa entre tais valores (log rank, p = 0,63; Figura 3).

As estimativas de salvamento de membro também não variaram significativamente entre as derivações: AFC, 83,0\%; AFS, 82,0\%; e AFP, 92,0\%, respectivamente, com EP < 10\% ( $\mathrm{p}=0,78$; Figura 4). 
Tabela 2 - Características técnicas dos procedimentos cirúrgicos

\begin{tabular}{|c|c|}
\hline Fatores & n (\%) \\
\hline \multicolumn{2}{|l|}{ Sítio da anastomose proximal } \\
\hline Femoral comum & $40(31,0)$ \\
\hline Femoral superficial & $72(55,8)$ \\
\hline Femoral profunda & $17(13,2)$ \\
\hline \multicolumn{2}{|l|}{ Sítio da anastomose distal } \\
\hline Poplítea abaixo do joelho & $33(25,6)$ \\
\hline Tronco tibioperônio & $6(4,7)$ \\
\hline Tibial anterior & $18(14,0)$ \\
\hline Tibial posterior & $28(21,7)$ \\
\hline Fibular & $34(26,4)$ \\
\hline Dorsal do pé & $5(3,9)$ \\
\hline Plantar comum & $1(0,8)$ \\
\hline Artérias geniculares & $1(0,8)$ \\
\hline Enxerto pérvio (sítio infragenicular) & $2(1,6)$ \\
\hline Társica lateral & $1(0,8)$ \\
\hline \multicolumn{2}{|l|}{ Substituto arterial } \\
\hline Veia safena devalvulada & $74(57,4)$ \\
\hline Veia safena reversa & $32(24,8)$ \\
\hline Veia safena in situ & $1(0,8)$ \\
\hline Veia safena parva & $2(1,6)$ \\
\hline Veia cefálica & $6(4,7)$ \\
\hline Veia basílica & $4(3,1)$ \\
\hline $\begin{array}{l}\text { Artéria femoral superficial endarterec } \\
\text { safena devalvulada }\end{array}$ & $1(0,8)$ \\
\hline Veias cefálica + safena devalvuladas & $3(2,3)$ \\
\hline Veias basílica + cefálica devalvuladas & $6(4,7)$ \\
\hline
\end{tabular}

A sobrevida do grupo em 36 meses foi de 64,4\%. Dezesseis pacientes morreram no período de internação, enquanto 27 indivíduos tiveram óbito no período de seguimento.

A estimativa de seguimento dos pacientes no período foi de $89 \%$ no grupo total, com a seguinte distribuição para os subgrupos: AFC, 91,0\%; AFS, 89,0\%; e AFP, $100,0 \%$.

No grupo das derivações com origem na AFP (17), ocorreram quatro oclusões de enxerto, duas revisões das derivações e duas grandes amputações, com estimativas de perviedade primária de $64,7 \%$, perviedade primária assistida de $70,6 \%$ e salvamento do membro de $92,0 \%$.

No programa de vigilância, nenhum enxerto com risco de oclusão foi detectado por lesão em seu corpo no período de seguimento. Entretanto, dois casos foram detectados com alterações de fluxo hemodinamicamente significativas: um caso no pós-operatório precoce, com escoamento proximal do enxerto por oclusão de um pequeno segmento juxta-anastomótico distal da artéria genicular sural, que foi submetido à extensão do enxerto com veia basílica; no outro caso, detecção de 


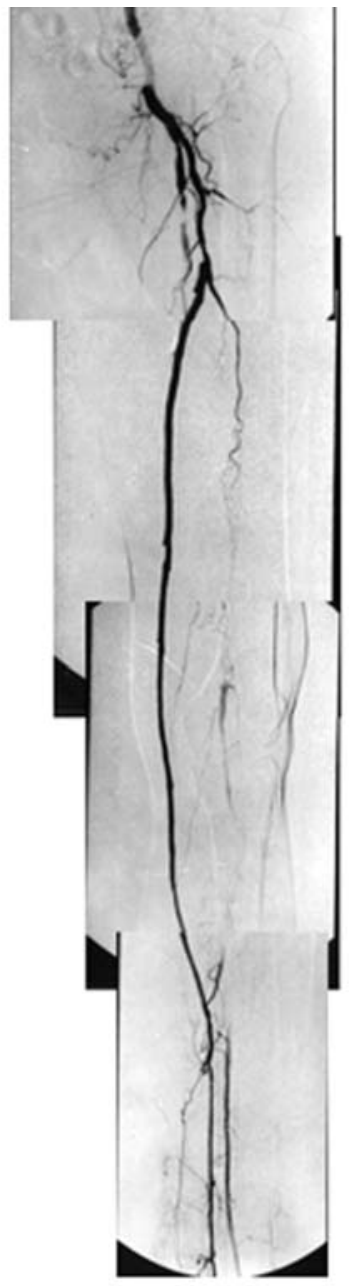

Figura 1 - Derivação da artéria femoral profunda distal (terceira porção) para a artéria tibial posterior com veia safena interna devalvulada, pelo acesso póstero-medial

estenose proximal significativa, que provocou a necessidade de revisão do fluxo doador, onde foi realizado enxerto da ilíaca externa (enxerto prévio, segunda porção da AFP fibular) com artéria femoral superficial endarterectomizada, com sucesso até o final do período de estudo. Essas revisões provocaram uma melhora de perviedade primária para primária assistida nas variáveis clínicas artérias infrapoplíteas, veias de braço, porções proximal e distal da AFP, cirurgias primária e secundária e lesão trófica, mas sem diferença estatística significante (Tabela 3).

Os enxertos com anastomose proximal na AFP apresentaram uma melhor perviedade quando implantados na artéria poplítea distal $(80,0 \% ; \mathrm{EP}=17 \%)$ em relação

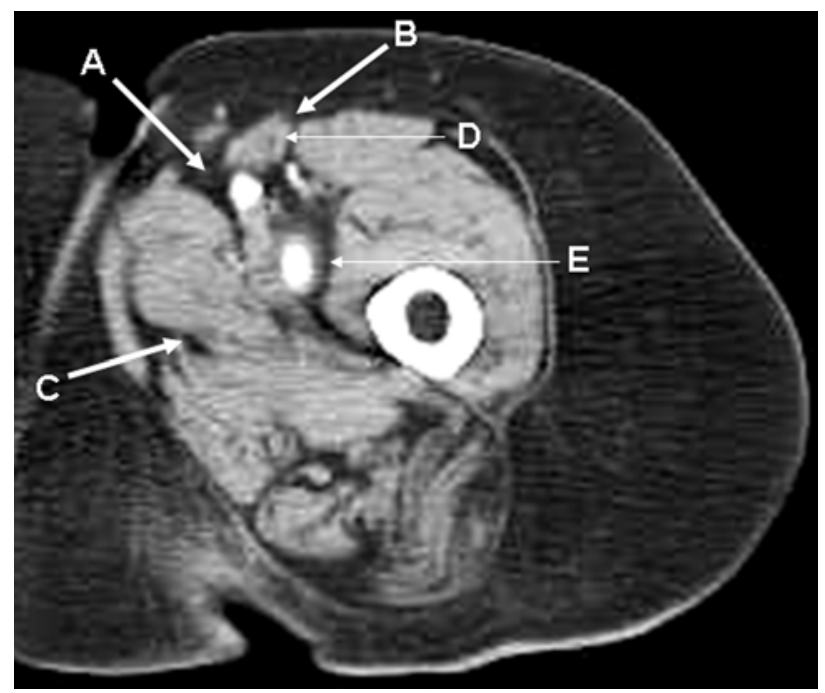

Figura 2 - Acessos A) ântero-medial, B) ântero-lateral e C) póstero-medial à artéria femoral profunda; D) músculo sartório identificado; E) artéria femoral profunda

às artérias infrapoplíteas $(50,0 \% ; \mathrm{EP}=14 \%)$, mas sem diferença estatística significante $(p=0,29)$.

As VMS presentes em 11 dos 17 enxertos da AFP mostraram estimativa de perviedade $(54,5 \% ; \mathrm{EP}=15 \%)$ equivalente à safena interna $(60,0 \% ; \mathrm{EP}=21 \%)$, sem diferença estatística significante $(\mathrm{p}=0,89)$.

Os cinco enxertos com origem na porção distal mostraram melhor estimativa de perviedade primária $(60,0 \% ; \mathrm{EP}=21 \%)$ que os 12 enxertos da primeira e segunda porções $(58,3 \%$; EP $=14 \%)$, mas sem diferença estatística significante $(\mathrm{p}=0,89)$.

A indicação cirúrgica por dor de repouso ou lesão trófica não influenciou as estimativas de perviedade primária dos enxertos $(54,5 \%, \mathrm{EP}=19 \%$ e $66,7 \%, \mathrm{EP}=$ $13 \%$, respectivamente), sem diferenças significantes estatisticamente $(\mathrm{p}=0,67)$.

$\mathrm{Na}$ avaliação da perviedade dos enxertos das cirurgias secundárias $(62,5 \%$; $\mathrm{EP}=15 \%)$ ou primárias $(53,3 \%$; EP $=15 \%)$, não houve diferença estatisticamente significante $(\mathrm{p}=0,77)$.

Na evolução da cicatriz cirúrgica dos acessos anteriores da primeira e segunda porções e do acesso pósteromedial da terceira porção, observamos hiperemia das cicatrizes cirúrgicas em $20 \%$ dos casos, mas não foram 


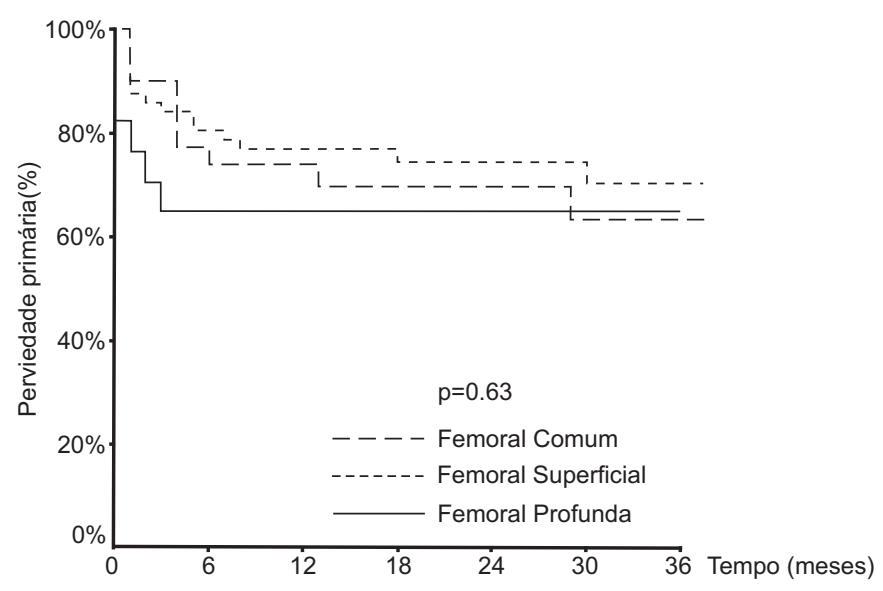

$\begin{array}{lllllllll}\text { Femoral comum } & \text { Em risco } & 40 & 23 & 19 & 15 & 11 & 10 & 09 \\ & \text { Erro padrão } & 0.000 & 0.076 & 0.076 & 0.082 & 0.082 & 0.096 & 0.096 \\ \text { Femoral superficial } & & & & & & & & \\ & \text { Em risco } & 72 & 45 & 36 & 28 & 25 & 19 & 16 \\ & \text { Erro padrão } & 0.000 & 0.048 & 0.053 & 0.058 & 0.058 & 0.066 & 0.066 \\ \text { Femoral profunda } & & & & & & & & \\ & \text { Em risco } & 17 & 11 & 10 & 08 & 07 & 07 & 07 \\ & \text { Erro padrão } & 0.000 & 0.115 & 0.115 & 0.115 & 0.115 & 0.115 & 0.115\end{array}$

Figura 3 - Perviedade primária das derivações infrageniculares estratificadas pelas diferentes artérias doadoras

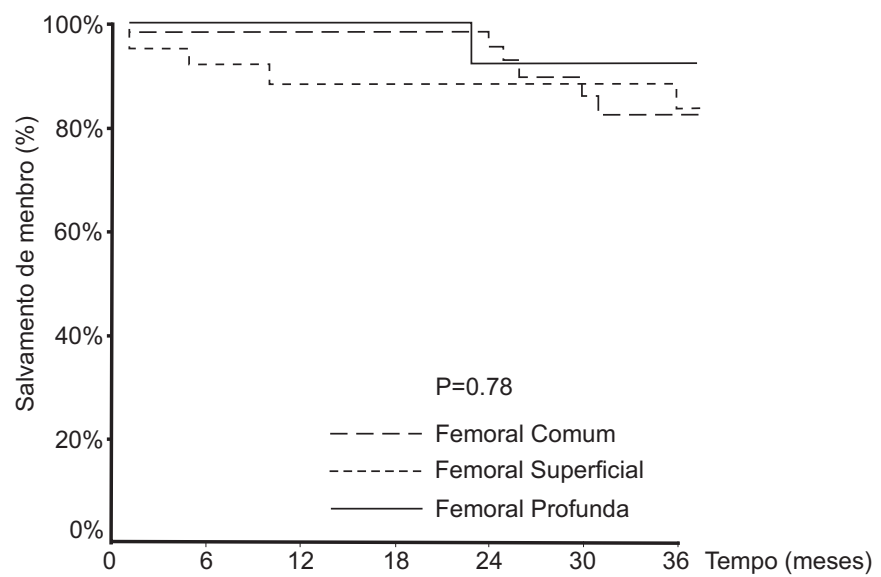

Femoral comum

$\begin{array}{llllllll}\text { Em risco } & 40 & 29 & 25 & 21 & 18 & 18 & 17\end{array}$

Femoral superficial

Erro padrão

$\begin{array}{lllllll}0.000 & 0.045 & 0.055 & 0.055 & 0.055 & 0.055 & 0.072\end{array}$

Em risco

$\begin{array}{lllllll}72 & 54 & 46 & 38 & 35 & 25 & 22\end{array}$

Erro padrão

$\begin{array}{lllllll}0.000 & 0.013 & 0.013 & 0.013 & 0.030 & 0.059 & 0.067\end{array}$

Femoral profunda

$\begin{array}{llllllll}\text { Em risco } & 17 & 15 & 14 & 13 & 12 & 12 & 12 \\ \text { Erro padrão } & 0.000 & 0.000 & 0.000 & 0.000 & 0.073 & 0.073 & 0.073\end{array}$

Figura 4 - Salvamento de membro das derivações infrageniculares estratificadas pelas diferentes artérias doadoras 
Tabela 3 - Perviedade primária e primária assistida dos enxertos da AFP conforme variáveis clínicas e técnicas

\begin{tabular}{|c|c|c|c|c|}
\hline Variável & $\begin{array}{c}\text { Perviedade primária em } \\
36 \text { meses }(\%)\end{array}$ & $\mathbf{p}$ & $\begin{array}{c}\text { Perviedade primária } \\
\text { assistida em } 36 \text { meses } \\
(\%)\end{array}$ & p \\
\hline \multicolumn{5}{|l|}{ Anastomose distal } \\
\hline $\begin{array}{c}\text { Poplítea } \\
\text { infragenicular }\end{array}$ & 80,0 & 0,29 & 80,0 & 0,56 \\
\hline $\begin{array}{l}\text { Artérias } \\
\text { infrapoplíteas }\end{array}$ & 50,0 & & 66,7 & \\
\hline \multicolumn{5}{|l|}{ Substituto arterial } \\
\hline Veia safena interna & 60,0 & 0,89 & 60,0 & 0,59 \\
\hline Veias de braço & 54,5 & & 72,7 & \\
\hline \multicolumn{5}{|l|}{ Segmento doador } \\
\hline Proximal & 58,3 & 0,89 & 66,7 & 0,61 \\
\hline Distal & 60,0 & & 80,0 & \\
\hline \multicolumn{5}{|l|}{ Cirurgia } \\
\hline Primária & 53,3 & 0,77 & 66,7 & 0,64 \\
\hline Secundária & 62,5 & & 75,0 & \\
\hline \multicolumn{5}{|l|}{ Quadro clínico } \\
\hline Lesão trófica & 54,5 & 0,67 & 72,3 & 0,83 \\
\hline Dor em repouso & 66,7 & & 66,7 & \\
\hline
\end{tabular}

registrados hematomas, necrose de bordas da ferida operatória ou queixa exagerada de dor no pós-operatório precoce.

\section{Discussão}

Autores como Farley et al. ${ }^{9}$, DePalma et al. ${ }^{4}$ e Aston et al. ${ }^{10}$ relataram o menor comprometimento aterosclerótico da AFP em relação à artéria femoral superficial nos casos de isquemia crítica, mostrando a sua potencial utilidade como doadora de fluxo de enxertos, tanto em cirurgias primárias quanto secundárias.

Beales et al. ${ }^{11}$ consideram que a projeção arteriográfica ântero-posterior subestima a estenose na origem da artéria femoral profunda e sugerem uma projeção oblíqua da região coxofemoral, para melhor avaliação da doença da AFP. Em nenhum desses casos, usamos o ecoDoppler arterial como estudo inicial para avaliação da AFP, embora em pacientes com restrição ao uso de meio de contraste iodado tenhamos indicado enxertos distais somente com a ultra-sonografia arterial ${ }^{12}$. Portanto, um bom estudo angiográfico é essencial para verificar se a AFP é apropriada para o uso.

Darling III et al. ${ }^{13}$ publicaram 563 casos mostrando que a AFP livre de doença proximal é tão útil quanto as artérias femorais, comum e superficial nas reconstruções arteriais com função satisfatória em 76\%. Utilizaram, na maioria das derivações distais, a técnica in situ e relataram dificuldades inerentes a essa técnica, como a calcificação da AFC ou oclusão da artéria femoral superficial, tornando difícil a anastomose proximal. Justificaram então que a utilização da porção média da AFP deixa a anastomose livre de tensão com bons resultados de função. Mills et al. ${ }^{2}$ relataram o uso da AFP nas porções medial e distal em 56 derivações arteriais, com resultado satisfatório de função secundária (96\%) em 36 meses, sendo a grande maioria pelo acesso anterior e, em somente dois casos, pelo acesso pósteromedial. No presente estudo, obteve-se perviedade primária assistida de $70,6 \%$. 
Mello et al. ${ }^{14}$, em sua extensa casuística de derivações infra-inguinais com veia safena interna in situ, mostraram que a artéria femoral profunda foi a segunda artéria mais utilizada como anastomose proximal de enxertos, constatando a freqüência de seu uso (23\%) e sua importância em situações alternativas. Mostraram bons resultados de perviedade dos enxertos no grupo total; entretanto, não analisaram especificamente a perviedade dos enxertos que tiveram anastomose proximal na artéria femoral profunda.

No presente estudo, observamos estimativas de perviedade primária melhores para os enxertos com origem nas artérias femorais, superficial $(70,2 \%)$ e profunda $(64,7 \%)$ em relação à artéria comum $(63,3 \%)$, mas sem diferença estatística significante ( $p=0,63$; Figura 3$)$, condição semelhante às publicações já citadas ${ }^{2,3,13}$.

No entanto, neste estudo, a AFP foi utilizada nas suas três porções, o que nos permitiu avaliar a execução e utilidade dos acessos, bem como seus resultados, em uma casuística ainda pequena, necessitando talvez de uma ampliação de sua indicação como doadora de fluxo, já que representou $13 \%$ dos enxertos totais, contra $23 \%$ do grupo de Mello et al. ${ }^{14}, 25 \%$ do grupo de Mills et al. ${ }^{2}$ e $24 \%$ do grupo de Darling III et al. ${ }^{13}$.

Existe uma preocupação quanto ao uso das porções distais da AFP devido à evolução natural da doença aterosclerótica. Darling III et al. ${ }^{13}$ relataram que 1,6\% dos casos precisaram de procedimentos proximais por doença hemodinamicamente significativa em lesões inicialmente não importantes, quando utilizaram a porção média da AFP. Em nossa casuística, encontramos somente um caso $(5 \%)$ de estenose proximal, que necessitou de revisão. Mills et al. ${ }^{2}$ relataram um único caso de revisão de artéria nativa proximal, fato que atribuíram à lesão por clampe e não à progressão da doença aterosclerótica. Essa preocupação diminui se analisarmos que a presença da hiperplasia intimal hemodinamicamente significativa detectada nos enxertos autógenos no primeiro ano de vigilância pelo duplex scan é de aproximadamente $25 \%{ }^{15,16}$, portanto maior que as taxas na progressão da doença, fato que nos permite tentar usar fontes de artérias mais distais e enxertos menos extensos.

Outra boa indicação para o uso das porções distais da AFP é a presença de prótese proximal no segmento aortoilíaco femoral, cuja exposição, sempre que possível, deve ser evitada devido ao maior risco de infecção, condição que também evita lesões em estruturas vizinhas (veias, nervos) durante a dissecção da área fibrosada.

Quanto à técnica de implante da veia nas derivações infrageniculares, estudos publicados por vários autores ${ }^{17-19}$ mostram que os resultados são semelhantes quando a equipe cirúrgica usa a técnica na qual tem maior experiência. Em nossa série, tivemos a oportunidade de utilizar as duas técnicas, conforme a preferência do cirurgião, e encontramos no grupo total melhor desempenho das veias safenas colocadas da maneira removida e devalvulada $(79,3 \%)$ quando comparadas com as reversas $(41,4 \%)$, resultados que apresentaram diferença estatisticamente significante $(\mathrm{p}<0,01)$.

O número de oclusões dos enxertos cuja anastomose proximal teve origem na primeira e segunda porções da AFP apresentou taxas equivalentes de falha aos que tiveram o implante proximal na porção distal, fato que provocou estimativas de perviedade primária equivalentes na utilização em qualquer porção da AFP disponível $(p=0,89)$.

A utilização da terceira porção da AFP pelo acesso póstero-medial foi relatada por Nunes et al. ${ }^{7}$ e mostrou bons resultados em cinco casos operados, com um seguimento de seis meses. Brochado-Neto et al. ${ }^{20}$ publicaram uma casuística de 11 enxertos com origem na porção distal, relatando bons resultados de função e preservação do membro.

O acesso póstero-medial possibilita o emprego de um segmento menos extenso de veia autógena, fato positivo para o desempenho do enxerto a curto e médio prazo, tanto em leitos distais com bom escoamento ${ }^{2,21}$ quanto em leitos com alta resistência periférica. No presente estudo, tivemos dois casos: um deles escoando para um segmento isolado de poplítea ${ }^{22-24} \mathrm{e}$ outro para uma artéria genicular sural ${ }^{25}$. O uso de segmentos selecionados de veia pode diminuir o risco de lesões preexistentes não percebidas no enxerto no momento da cirurgia ${ }^{26}$.

Logerfo et al. ${ }^{27}$ descreveram a técnica de utilização em alça das veias cefálica e basílica ligadas pela veia mediana do cotovelo em segmento único, condição que 
permite um melhor aproveitamento em extensão da VMS, e que utilizamos em quatro casos satisfatoriamente.

Não empregamos prótese como substituto, evitando a colocação de material sintético próximo às áreas de infecção ou em reoperações, porque preferimos o material autógeno, como as VMS, que sabidamente tem melhores resultados de função em enxertos distais do que a prótese ${ }^{28-31}$.

As cirurgias primárias e secundárias apresentaram resultados satisfatórios e equivalentes, em consonância com as publicações de Whittemore et al. ${ }^{32}$ e Belkin et al. ${ }^{33}$, que relataram a importância das reoperações no tratamento da isquemia crítica.

A vigilância dos enxertos autógenos conforme recomendado pelo TASC $^{34}$ provocou duas revisões de enxertos, que foram realizadas com sucesso, melhorando a perviedade primária assistida, situação que é respaldada por publicações que relatam melhores resultados das revisões de enxertos em relação às trombectomias de enxertos ou reoperações precoces ${ }^{35-37}$.

Sabemos das preocupações dos cirurgiões na abordagem da AFP. Entretanto, atualmente, as publicações mostram resultados equivalentes de função da AFP a outras artérias doadoras de fluxo para enxertos, e tendo em vista a pequena taxa de progressão da doença aterosclerótica proximal, o aproveitamento da AFP poderia ser realizado com maior freqüência, nas suas três porções, mesmo em cirurgias primárias, como, por exemplo, o abdome em avental, condição que freqüentemente escarifica a prega inguinal e é foco de infecção da ferida cirúrgica.

\section{Conclusão}

Os resultados deste estudo mostraram bom desempenho da AFP como doadora de enxerto nas suas três porções nas variáveis de desfecho analisadas. Mostraram também que sua utilização é segura e viável nas várias situações desfavoráveis aos acessos convencionais para origem de enxertos distais.

\section{Referências}

1. Veith FJ, Gupta SK, Samson RH, Flores SW, Janko G, Scher LA. Superficial femoral and popliteal arteries as inflow sites for distal bypasses. Surgery. 1981;90:980-90.
2. Mills LM, Taylor SM, Fujitani RM. The role of the deep femoral artery as an inflow site for infrainguinal revascularization. J Vasc Surg. 1993;18:416-23.

3. Ballotta E, Renon L, De Rossi A, Barbon B, Terranova O, Da Giau G. Prospective randomized study on reversed saphenous vein infrapopliteal bypass to treat limb-threatening ischemia: Common femoral artery versus superficial femoral or popliteal and tibial arteries as inflow. J Vasc Surg. 2004;40:732-40.

4. DePalma RG, Malgieri JJ, Rhodes RS, Clowes AW. Profunda bypass for secondary revascularization. Surg Gynecol Obstet. 1980;151:387-90.

5. Harris RW, Andros G, Dulawa LB, Oblath RW, Salles-Cunha SX, Apyan R. Successful long-term limb salvage using cephalic vein bypass grafts. Ann Surg. 1984;200:785-92.

6. Chang BB, Paty PS, Shah DM, Leather RP. The lesser saphenous vein? An underappreciated source of autogenous vein. J Vasc Surg. 1992;15:152-6; discussion 156-7.

7. Nunez AA, Veith FJ, Collier P, Ascer E, Flores SW, Gupta SK. Direct approaches to the distal portions of the profunda femoris artery for limb salvage bypasses. J Vasc Surg. 1988;8:576-8.

8. Rutherford RB. Atlas of vascular surgery: basic technics and exposures. Philadelphia: WB Saunders; 1993. p. 130-1.

9. Farley HH, Kiser JC, Hitchcock CR. Profunda femorispopliteal shunt. Ann Surg. 1964;160:23-5.

10. Aston NO, Thomas ML, Burnand KJ. The distribution of atherosclerotic in lower limbs. Eur J Vasc Surg. 1992;6:73-7.

11. Beales JS, Adcock FA, Frawley JS, et al. The radiological assessment of disease of the profunda femoris artery. Br J Radiol. 1971;44:854-9.

12. Mazzariol F, Ascher E, Hingorani A, Gunduz Y, Yorkovich W, Salles-Cunha S. Lower-extremity revascularisation without preoperative contrast arteriography in 185 cases: lessons learned with duplex ultrasound arterial mapping. Eur J Vasc Endovasc Surg. 2000;19:509-15.

13. Darling RC 3rd, Shah DM, Change BB, Lloyd WE, Leather RP. Can the deep femoral artery be used reliably as an inflow source for infrainguinal reconstruction? Long- term results in 563 procedures. J Vasc Surg. 1994;20:889-94; discussion 894-5.

14. Vieira de Mello A, Torres dos Santos CM, Oliveira BC, Vieira de Mello R. Revascularização distal dos membros inferiores: experiência de 13 anos. J Vasc Bras. 2002;1:181-92.

15. Donaldson MC, Mannick JA, Whittemore AD. Causes of primary failure after in situ saphenous vein bypass graft. J Vasc Surg. 1992;15:113-8; discussion 118-20.

16. Idu MM, Blankestein JD, de Gier P, Truyen E, Buth J. Impact of a color-flow duplex surveillance program on infrainguinal vein graft patency: a five-year experience. J Vasc Surg. 1993; 17:42-52.

17. Belkin M, Knox J, Donaldson MC, Mannick JA, Whittemore AD. Infrainguinal arterial reconstruction with nonreversed greater saphenous vein. J Vasc Surg. 1996;24:957-62.

18. Mills NL, Ochsner JL. Valvulotomy of valves in saphenous vein grafts before coronary artery bypass. J Thorac Cardiovasc Surg. 1976;71:878-9. 
19. Taylor LM Jr., Edwards JM, Porter JM. Present status of reversed vein bypass grafting: five years results of a modern series. J Vasc Surg. 1990;11:193-205; discussion 205-6.

20. Brochado-Neto FC, Albers M, Romiti M. The distal zone of the deep artery as the inflow site in femorodistal bypass grafting. Eur J Vasc Endovasc Surg. 2006;31:407-9.

21. Shah DM, Darling RC 3rd, Chang BB, Bock DE, Leather RP. Durability of short bypasses to infragenicular arteries. Eur J Vasc Endovasc Surg. 1995;10:440-4.

22. Kram HB, Gupta SK, Veith FJ, Wengerter KR, Panetta TF, Nwosisi C. Late results of two hundred seventeen femoropopliteal bypasses to isolated popliteal artery segments. J Vasc Surg. 1991;14:386-90.

23. Ascer E, Veith FJ, Gupta SK, et al. Short vein graft: A superior option for arterial reconstructions to poor or compromised outflow tracts? J Vasc Surg. 1988;7:370-8.

24. Belkin M, Welch HJ, Mackey WC, O`Donnell TF Jr. Clinical and hemodynamic results of bypass to isolated tibial artery segments for ischemic ulceration of the foot. Am J Surg. 1992;164:281-4; discussion 284-5.

25. Brochado Neto FC, Gonzalez J, Cinelli M Jr., Albers M. Bypass to the genicular arteries for revascularisation of the lower limb. Eur J Vasc Endovasc Surg. 2000;20:545-9.

26. Panetta TF, Marin ML, Veith FJ, et al. Unsuspected preexisting saphenous vein disease: an unrecognized cause of vein bypass failure. J Vasc Surg. 1992;15:102-10; discussion 110-2.

27. LoGerfo FW, Paniszyn CW, Menzoian J. A new arm vein graft for distal bypass. J Vasc Surg. 1987;5:889-91.

28. Faries PL, Arora S, Pomposelli FB Jr., et al. The use of arm vein in lower Extremity revascularization: Results of 520 procedures Performed in eight years. J Vasc Surg. 2000;31(1 Pt 1):50-9.

29. Harward TR, Coe D, Flynn TC, Seeger JM. The use of arm vein conduits during infrageniculate arterial bypass. J Vasc Surg. 1992;16:420-6; discussion 426-7.
30. Brochado-Neto FC, Albers M, Pereira CA, Gonzalez J, Cinelli M Jr. Prospective comparison of arm veins and greater saphenous veins as infrageniculate bypass grafts. Eur J Vasc Endovasc Surg. 2001;22:146-51.

31. Londrey GL, Bosher LP, Brow PW, Stoneburner FD Jr., Pancoast JW, Davis RK. Infrainguinal reconstruction with arm vein, lesser saphenous vein, and remnants of greater saphenous vein: a report of 257 cases. J Vasc Surg. 1994;20:451-7.

32. Whittemore AD, Clowes AW, Couch NP, Mannick JA. Secondary femoropopliteal reconstruction. Ann Surg. 1981;193:3542.

33. Belkin M, Conte MS, Donaldson MC, Mannick JA, Whittemore AD. Preferred strategies for secondary infrainguinal bypass: lesons learned from 300 consecutive reoperations. J Vasc Surg. 1995;21:282-93; discussion 293-5.

34. TransAtlantic InterSociety Consensus (TASC). Management of peripheral arterial disease: Surveillance after revascularization. J Vasc Surg. 2000;31(Suppl 2):S253-60.

35. Berkowitz HD, Fox AD, Deaton DH. Reversed vein graft stenosis: Early diagnosis and management. J Vasc Surg. 1992;15:130-41; discussion 141-2.

36. Bandyk DF, Bergamini TM, Towne JB, Schimitt DD, Seabrook GR. Durability of vein graft revision: The outcome of secondary procedures. J Vasc Surg. 1991;13:200-8; discussion 209-10.

37. Darling RC 3rd, Roddy SP, Chang BB, et al. Long-term results of revised infrainguinal arterial reconstructions. J Vasc Surg. 2002;35:773-8.

Correspondência:

Francisco Cardoso Brochado Neto

Rua Canário, 111/62B, Moema

CEP 04521-005 - São Paulo, SP

E-mail: francardnet@terra.com.br, cirurgiavascularhspe@yahoo.com.br 